\title{
A New Client/Server Software Platform for Efficient Remote Microscope Acquisition, Control, Automation and Analysis
}

\author{
J. K. Weiss and W. J. de Ruijter \\ Emispec, 2050 S. Cottonwood Dr., Tempe, AZ 85282
}

A need for remote microscope control has existed for many years, and is driven by several factors. These factors include the desires to share expensive equipment with users in other facilities, to interact with remote collaborators during experiments, to physically separate the operator from high-resolution instrumentation in order to improve performance, and to access from any location the data acquired during a microscope session. Most early attempts at remote operation centered on so-called "telepresence microscopy" which used a combination of video conferencing, remote computer desktops, and custom control panels to reflect some local control to a remote client [1,2]. More recent efforts have used some remote calling protocols to allow for some control and acquisition from remote hardware [3]. We have developed Cynapse, a new client/server software platform that enables highly efficient remote operation that in most cases is identical to local operation. The platform also enables distributed access to data acquired from the instrument.

The key components of the platform are shown in Fig. 1. All control and acquisition is done by the Instrument Server computer that is local to the instrument. The experimental algorithms run in the server logic on the Instrument Server, which eliminates the need for client-server roundtrips when feedback to the microscope is required during the experiment. All persistent data generated by the server logic is stored in a database that is accessible to both the server and the client logic. The client both receives and displays new data pushed from the server, and also translates user interface input events into commands that are sent to the server logic.

In this architecture, bandwidth limitations are minimized, because the vast majority of the dataintensive operations are carried out on the server. In general, it is only necessary to transmit a rendered image (typically 8-bit depth), which can also be compressed with varying quality depending on the viewing rate. Table 1 shows typical network bandwidth load for several remoting experiments. The bandwidth requirement for Internet remoting is lower because of the use of higher data compression, resulting in somewhat lower quality for images, which use lossy compression algorithms. This makes it possible to view high frame rate imaging such as video, even over the Internet. For lower frame rate image viewing we have implemented server logic for automating all key microscope adjustments in order to reduce the need for real-time feedback. Available automation functions for viewing and basic alignment are listed in Table 2.

Using the server-side automation capabilities of this new platform, we have produced a range of sophisticated experiments, such as drift-corrected multi-spectroscopy spectrum imaging and STEM electron tomography, all of which can be run remotely. On the client side, it is possible to process the acquired data offline using the experiment data stored in the database, or even to offload the data into enterprise database systems, such as LIMS systems, for distributed archiving and retrieval. Finally, because of the client/server separation, it is possible to provide a single client access point to multiple instruments in a facility, with a single user interface which integrates all functionality.

\section{References}

[1] N. J. Zaluzec, Microscopy \& Microanalysis 6 (2000) 1178.

[2] M. Hadida-Hassan et al., J. Struct. Biology 125 (1999) 235.

[3] K. Fukishima et al., Microscopy \& Microanalysis 6 (2000) 1144. 


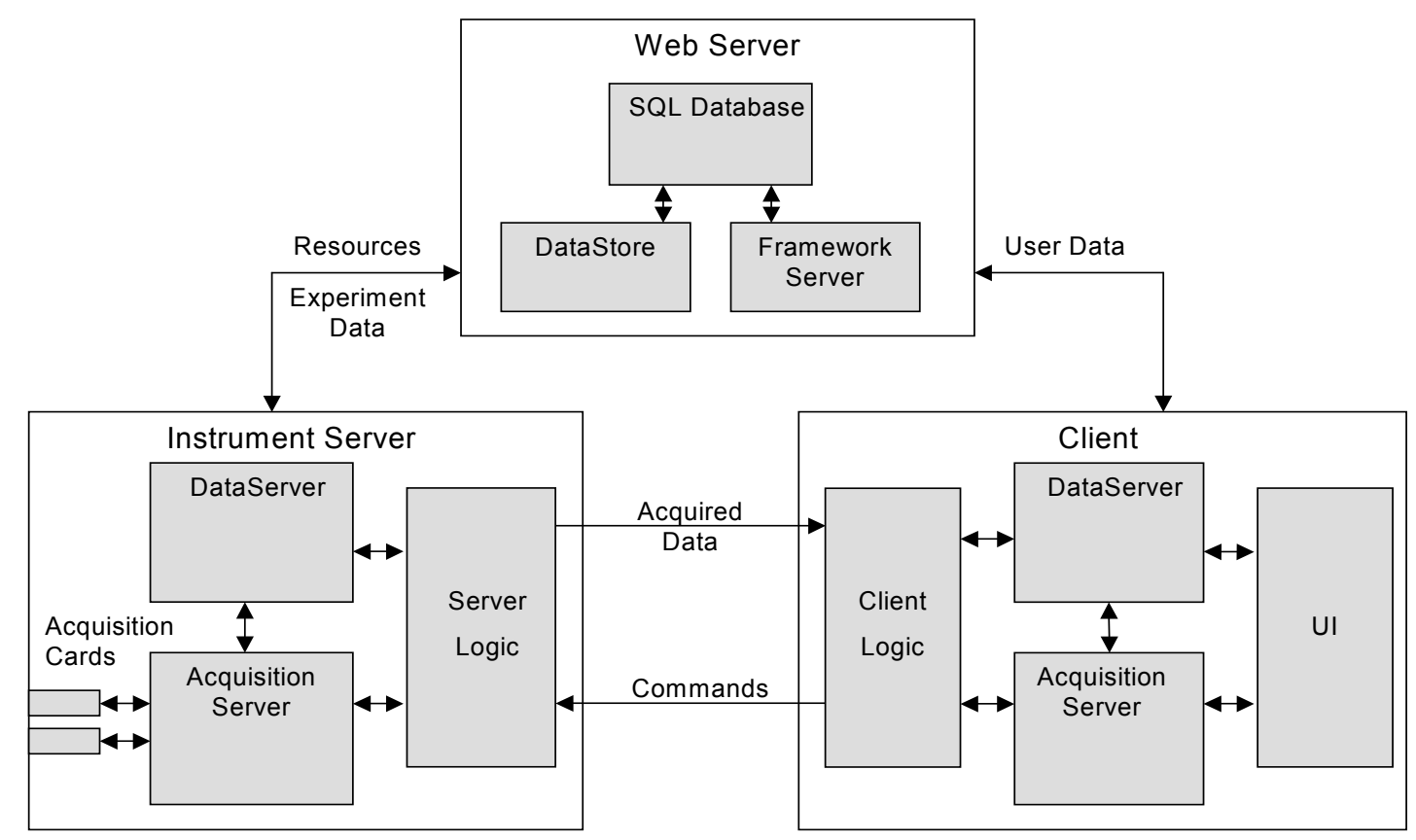

FIG. 1. Block architecture for the Cynapse platform showing key components for remoting.

TABLE 1. Typical Internet and Intranet bandwidths used by several remote experiments.

\begin{tabular}{lcc}
\hline \multicolumn{1}{c}{ Experiment } & $\begin{array}{c}\text { Intranet Bandwidth } \\
(\mathrm{kB} / \mathrm{sec})\end{array}$ & $\begin{array}{c}\text { Internet Bandwidth } \\
(\mathrm{kB} / \mathrm{sec})\end{array}$ \\
\hline CCD View $(512 \times 512-2$ frames/sec) & 500 & 40 \\
\hline EELS View $(5$ spectra/sec) & 25 & 15 \\
\hline Live STEM FFT $(500 \times 500-2$ frames/sec) & 1000 & 60 \\
\hline CCD Tomography w/Tracking & 200 & 20 \\
\hline Video View (512x512-15 frames/sec) & 2000 & 100 \\
\hline
\end{tabular}

TABLE 2. Available adjustment experiments for remote viewing, with typical execution time.

\begin{tabular}{llc}
\hline \multicolumn{1}{c}{ Experiment } & \multicolumn{1}{c}{ Description } & Time (sec) \\
& & 3 \\
\hline TEM Focus & Autofocus using beam tilt-induced shift & 5 \\
\hline TEM Stigmation & Auto stigmation using beam tilt-induced shift & 2 \\
\hline TEM Brightness & Auto brightness adjust using condenser lenses & 4 \\
\hline STEM Focus & Autofocus using image power spectrum monitoring & 2 \\
\hline STEM ACB & Auto contrast/brightness using STEM amplifiers & 3 \\
\hline Beam alignment & On-axis adjustment of beam tilt & 10 \\
\hline Eucentric height & Auto adjustment of specimen height & $0.5-2.0$ \\
\hline Specimen center & Click-to-center function using image/stage shift & \\
\hline
\end{tabular}

